\title{
On the Diversity-Multiplexing Tradeoff of Cooperative Multicast System with Wireless Network Coding
}

\author{
Jun Li and Wen Chen
}

\begin{abstract}
Diversity-multiplexing tradeoff (DMT) is an efficient tool to measure the performance of multiple-input and multiple-output (MIMO) systems and cooperative systems. Recently, cooperative multicast system with wireless network coding stretched tremendous interesting due to that it can drastically enhance the throughput of the wireless networks. It is desirable to apply DMT to the performance analysis on the multicast system with wireless network coding. In this paper, DMT is performed at the three proposed wireless network coding protocols, i.e., non-regenerative network coding (NRNC), regenerative complex field network coding (RCNC) and regenerative Galois field network coding (RGNC). The DMT analysis shows that under the same system performance, i.e., the same diversity gain, all the three network coding protocols outperform the traditional transmission scheme without network coding in terms of multiplexing gain. Our DMT analysis also exhibits the trends of the three network coding protocols' performance when multiplexing gain is changing from the lower region to the higher region. Monte-Carlo simulations verify the prediction of DMT.
\end{abstract}

Index Terms: Cooperative communications, diversity-multiplexing tradeoff (DMT), wireless multicast systems, wireless network coding.

\section{INTRODUCTION}

Network coding has been proven to achieve the network multicast capacity bound in wired networks [1]. Since network coding can enhance the network throughput, there have been a lot of research works on different coding approaches [2]-[5]. Recently, how to leverage network coding in wireless networks for system capacity improvement has drawn increasing interest [6][13]. Different from wired networks, wireless transmissions occurring in the air must unfortunately face many disadvantageous factors such as channel fading, noises and broadcast interferences. Till now, there is no a good theoretic metric to measure the performance of a wireless network coding protocols. In this paper, diversity-multiplexing tradeoff (DMT) formulation is applied to the proposed wireless network coding protocols.

The DMT is first put forward for multiple-input and multipleoutput (MIMO) systems [14], in which Zheng and Tse give a

Manuscript received July 17, 2008; approved for publication by Tracey Ho, Guest Editor, March 09, 2009.

This work is supported by NSF China \#60972031, by SEU-SKL open project \#W200907, by National 973 project \#2009CB824900, by Cultivation Fund of the Key Scientific and Technical Innovation project, Ministry of Education of China \#706022, by program for New Century Excellent Talents in University \#NCET-06-0386, and by Huawei Funding \#YJCB2008048WL and \#YJCB2009024WL.

The authors are with Department of Electronic Engineering, Shanghai Jiao Tong University, Shanghai, 200240, China. W. Chen is also with the SKL for Mobile Communications, Southeast University, China, email: \{jleesr80, wenchen\}@sjtu.edu.cn.

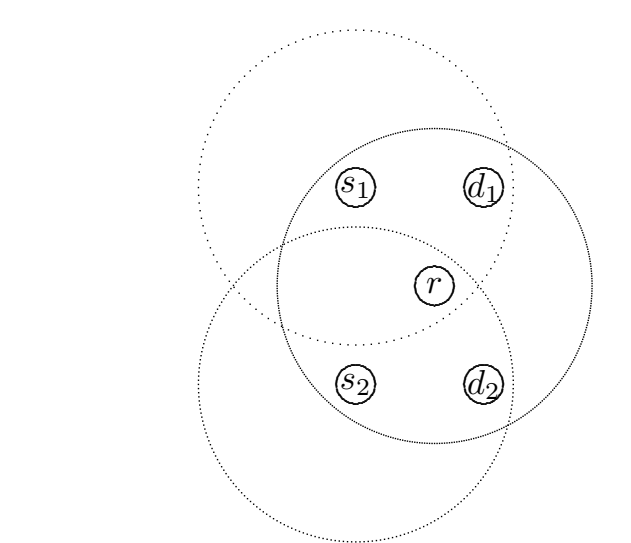

Fig. 1. A wireless $2-1-2$ multicast system where the circles denote the transmission ranges. Due to the power constraint, $s_{1}\left(s_{2}\right)$ should borrow $r$ to transmit messages to $d_{2}\left(d_{1}\right)$.

formulation between the diversity gain and multiplexing gain by using the Gaussian code. The DMT assumes a family of codes, in which diversity gain $d$ and multiplexing gain $\lambda$ are defined by

$$
d \triangleq-\lim _{\rho \rightarrow \infty} \frac{\log \left(P_{e}(\rho)\right)}{\log \rho} \quad \text { and } \quad \lambda \triangleq \lim _{\rho \rightarrow \infty} \frac{R(\rho)}{\log \rho}
$$

where $\rho, P_{e}(\rho)$, and $R(\rho)$ represent the signal-to-noise ratio (SNR), frame error probability (FEP) and transmission rate, respectively. So a scheme's DMT means that at the $\lambda$ multiplexing gain, the diversity gain that the scheme obtains should not exceed $d(\lambda)$. Now, this elegant formulation is successfully used as a standard in cooperative communications to evaluate the performance of different transmission protocols [15]-[21].

On the other hand, in [6]-[10], wireless network coding is applied to bi-directional traffic flows or multi-access channels to increase the system capacity. However, these applications are not suitable to more complicated wireless networks which are always composed of multicast cells. Specifically, we consider a basic multicast model with two sources, two destinations and one half-duplex constrained relay $(2-1-2)$ wireless multicast network [13], [22]. In Fig. 1, we suppose that $s_{1}$ as well as $s_{2}$ broadcast their information to the two destinations $d_{1}$ and $d_{2}$ simultaneously. From Fig. 1, we can see $d_{1}$ (or $d_{2}$ ) is out of the transmission range of $s_{2}$ (or $s_{1}$ ). The shared relay can help $s_{1}$ and $s_{2}$ reach their destinations. There are two transmission schemes. The first scheme is by the traditional way without network coding, which occupies four time slots:

1. $s_{1} \rightarrow\left\{r, d_{1}\right\}$ with information $I_{s_{1}}$;

2. $r \rightarrow d_{2}$ with information $I_{s_{1}}$;

3. $s_{2} \rightarrow\left\{r, d_{2}\right\}$ with information $I_{s_{2}}$;

4. $r \rightarrow d_{1}$ with information $I_{s_{2}}$.

The second one is by wireless network coding method that is 
more efficient:

1. $s_{1} \rightarrow\left\{r, d_{1}\right\}$ with information $I_{s_{1}}$,

$s_{2} \rightarrow\left\{r, d_{2}\right\}$ with information $I_{s_{2}}$;

2. $r \rightarrow\left\{d_{1}, d_{2}\right\}$ with $f\left(I_{s_{1}}, I_{s_{2}}\right)$.

For the network coding scheme, because $d_{1}$ (or $d_{2}$ ) has already detected the information $I_{s_{1}}$ (or $I_{s_{2}}$ ) in the first time slot, it can easily pick up the remained information $I_{s_{2}}$ (or $I_{s_{1}}$ ) from the combined signals transmitted by $r$ in the second time slot. Function $f(\cdot)$ in the relay is certain operation, according to which, we define three network coding protocols, i.e., nonregenerative network coding (NRNC) where the relay broadcasts the mixed signals from sources to the destinations without decoding them, regenerative complex field network coding (RCNC) where relay decodes the mixed signals and then superposes the decoded symbols in complex field before retransmission, and regenerative Galois field network coding (RGNC) where the decoded symbols are superposed in Galois field before retransmission. Note that in RGNC, the decoded symbols are demodulated into bit streams before superposition in $\mathrm{Ga}$ lois field, following which, the combined new bit stream is then modulated to certain symbol and transmitted.

The notation used in this paper goes as follows. $(x)^{+}$denotes $\max \{0, x\},(x)^{-}$denotes $\min \{0, x\}$, and $R^{N}$ and $C^{N}$ denote the set of real and complex $N$-tuples, respectively. $R^{N+}$ denotes the set of non-negative $N$-tuples. If some set $O \subseteq R^{N}$, we denote the complementary set of $O$ as $O^{c}$, while $O \cap R^{N+}$ as $\mathrm{O}^{+} . \boldsymbol{\Lambda}_{\mathrm{x}}$ denotes the auto-covariance matrix of vector $\mathbf{x}$.

\section{SYSTEM MODEL AND PRELIMINARIES}

In this section, we will set up the system model and make some preliminaries.

\section{A. System Model}

We setup the $2-1-2$ multicast system where channel coefficients shown in Fig. 2 are assumed to be flat fading with i.i.d. zero-mean, circularly symmetric complex Gaussian distribution and quasi-static in at least one frame period. So their magnitudes are Rayleigh distributed. The noises observed by all receivers are assumed to have a Gaussian distribution with zero mean and variance $\sigma^{2}$. Note that joint maximum likelihood (ML) decoding is performed at all the receivers.

We denote $P$ as the average total network transmission power over a time slot. Then, the system SNR is defined as $\rho \triangleq \frac{P}{\sigma^{2}}$. Since each destination should receive the signals from the both sources, we define the system frame as $\mathbf{x}_{s} \triangleq$ $\left[x_{s_{1}, 1}, \cdots, x_{s_{1}, N}, x_{s_{2}, 1}, \cdots, x_{s_{2}, N}\right]^{T}$ which is composed of the symbols from two sources, i.e., $\mathbf{x}_{s_{k}}=\left[x_{s_{k}, 1}, x_{s_{k}, 2}, \cdots, x_{s_{k}, N}\right]^{T}$ for the $k$ th source $s_{k}(k=1,2)$. All the symbols in $\mathbf{x}_{s}$ are assumed to be equally probable from the same $2^{R}$-QAM constellation set $Q$ with zero mean and variance $2 P$, where $R$ is the transmission rate. In the two regenerative network coding protocols, the decoded frame in the relay is denoted as $\mathbf{x}_{r} \triangleq\left[\mathbf{x}_{r_{1}}, \mathbf{x}_{r_{2}}\right]^{T}$ where $\mathbf{x}_{r_{k}}=\left[x_{r_{k}, 1}, \cdots, x_{r_{k}, N}\right]^{T}$. If the relay can successfully decode $\mathbf{x}_{s}$, we have $\mathbf{x}_{s_{k}}=\mathbf{x}_{r_{k}}$ and $\mathbf{x}_{s}=$ $\mathbf{x}_{r}$. The signals received in the $k$ th destination $d_{k}$ are denoted as $\mathbf{y}_{d_{k}}=\left[y_{d_{k}, 1}, y_{d_{k}, 2}, \cdots, y_{d_{k}, 2 N}\right]^{T}$.
The transmission scheme of the system just follow the second scheduling scheme [16] due to the half-duplex constraint of the relay, where a system frame period contains $2 N$ time slots, i.e., in the first $N$ time slots, both sources transmit $N$ symbols simultaneously, $d_{k}$ only receives the signals from $s_{k}$ and the relay receives the interferential signals of the sources which is mixed in the air, while in the remained $N$ time slots, the relay broadcasts the network coded signals to both destinations. Although the power consumption during a frame period is $2 N P$, it is necessary to further discuss the power allocation schemes (PAS) between all transmitters including the two sources and the relay. We denote $\kappa_{1}, \kappa_{2}$, and $\tau$ as power allocation factors (PAF) for $s_{1}, s_{2}$, and $r$, respectively. Then, power constraint is assumed to satisfy $\kappa_{1}+\kappa_{2}+\tau=1$.

For the three network coding protocols, the signals received by the relay in the first $N$ time slots are the same, i.e.,

$$
y_{r, i}=g_{1} \sqrt{\kappa_{1}} x_{s_{1}, i}+g_{2} \sqrt{\kappa_{2}} x_{s_{2}, i}+v_{r, i}, \text { for } i=1, \cdots, N
$$

where $v_{r, i}$ is the noise observed by the relay in the $i$ th time slot. However, the signals received by the destinations are different between the three network coding protocols.

\section{A.1 NRNC Protocol}

According to the power allocation in each transmitter, we further define the amplification factor of the relay as $b=$ $\sqrt{2 \tau P /\left(2 \kappa_{1}\left|g_{1}\right|^{2} P+2 \kappa_{2}\left|g_{2}\right|^{2} P+\sigma^{2}\right)}$. The signals received by $d_{k}$ during a frame period are written as

$$
\begin{aligned}
& y_{d_{k}, i}=\hbar_{k} \sqrt{\kappa_{k}} x_{s_{k}, i}+v_{d_{k}, i}, \text { for } i=1, \cdots, N, \\
& y_{d_{k}, N+i}=b h_{k} y_{r, i}+v_{d_{k}, N+i}
\end{aligned}
$$

where $v_{d_{k}, i}$ are the noises observed by $d_{k}$ in the $i$ th time slot.

\section{A.2 RCNC Protocol}

Since joint ML decoder is applied to the relay after the first $N$ time slots, the decoded frame in the relay is $\mathbf{x}_{r}$. The signals to be transmitted by the relay is denoted as $\mathbf{x}_{r}^{c}$, which is obtained by the complex field superposition between $\mathbf{x}_{r_{1}}$ and $\mathbf{x}_{r_{2}}$. Denote $\tau_{k}$ as the PAF of the decoded symbols in $\mathbf{x}_{r_{k}}$ and we have $\mathbf{x}_{r}^{c}=$ $\sqrt{\tau_{1}} \mathbf{x}_{r_{1}}+\sqrt{\tau_{2}} \mathbf{x}_{r_{2}}$. Due to the power constraint at the relay, there is $\tau_{1}+\tau_{2}=\tau$. Then,

$$
\begin{aligned}
& y_{d_{k}, i}=\hbar_{k} \sqrt{\kappa_{k}} x_{s_{k}, i}+v_{d_{k}, i}, \text { for } i=1, \cdots, N, \\
& y_{d_{k}, N+i}=h_{k}\left(\sqrt{\tau_{1}} x_{r_{1}, i}+\alpha \sqrt{\tau_{2}} x_{r_{2}, i}\right)+v_{d_{k}, N+i}
\end{aligned}
$$

where $\alpha=e^{j \frac{3 \pi}{4}}$ is the precoder utilized to achieve the full diversity gain [7] and $x_{r_{k}, i}$ is the $i$ th decoded symbol of $s_{k}$.

\section{A.3 RGNC Protocol}

In RGNC, we denote the signals to be transmitted by the relay as $\mathbf{x}_{r}^{g}$, which is obtained by Galois field operation between $\mathbf{x}_{r_{1}}$ and $\mathbf{x}_{r_{2}}$. Since the two decoded symbols are combined into a new one, we have $\mathbf{x}_{r}^{g}=\left[x_{r, 1}, \cdots, x_{r, N}\right]^{T}$. Then, the signals received by $d_{k}$ are

$$
\begin{aligned}
& y_{d_{k}, i}=\hbar_{k} \sqrt{\kappa_{k}} x_{s_{k}, i}+v_{d_{k}, i}, \text { for } i=1, \cdots, N, \\
& y_{d_{k}, N+i}=h_{k} \sqrt{\tau} x_{r, i}+v_{d_{k}, N+i}
\end{aligned}
$$

where $x_{r, i} \in Q$ is the new symbol produced by the $i$ th decoded symbols $x_{r_{1}, i}$ and $x_{r_{2}, i}$ in Galois field. 


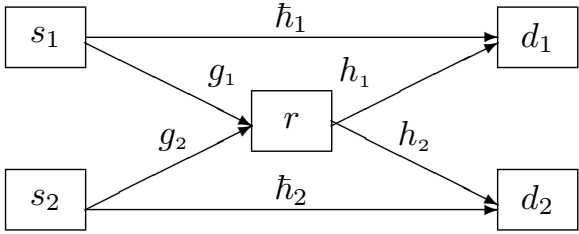

Fig. 2. $2-1-2$ multicast cooperative system.

\section{B. Preliminaries}

In the sequel, we follow [19] and summarize several expressions and results that may be used latter. We denote that as $\rho \rightarrow \infty$, i.e., $f(\rho) \doteq \rho^{a}$ if function $f(\rho)$ is exponentially equal to $\rho^{a}$,

$$
\lim _{\rho \rightarrow \infty} \frac{\log f(\rho)}{\log \rho}=a .
$$

Assume a Rayleigh channel coefficient $g$ with zero mean and unit variance and $z$ denote the order of $1 /|g|^{2}$. Then,

$$
z=-\lim _{\rho \rightarrow \infty} \frac{\log |g|^{2}}{\log \rho} .
$$

The probability density function (PDF) of $z$ can be shown as

$$
p_{z}=\lim _{\rho \rightarrow \infty} \ln (\rho) \rho^{-z} \exp \left(-\rho^{-z}\right) .
$$

The expression of (8) reveals that

$$
p_{z} \doteq \begin{cases}\rho^{-\infty}=0, & \text { for } \quad z<0, \\ \rho^{-z}, & \text { for } \quad z \geq 0 .\end{cases}
$$

Thus, for independent random variables $\left\{z_{i}\right\}_{i=1}^{N}$ distributed identically to $z$, the probability $P_{O}$ that $\left(z_{1}, \cdots, z_{N}\right)$ belongs to set $O$ can be characterized by

$$
P_{O} \doteq \rho^{-d_{o}} \quad \text { where } \quad d_{o} \triangleq \inf _{\left(z_{1}, \cdots, z_{N}\right) \in O^{+}} \sum_{i=1}^{N} z_{i}
$$

provided that $\mathrm{O}^{+}$is not empty, that is, the exponential order of $P_{O}$ only depends on $O^{+}$. This is because that the probability of any set, consisting of $N$-tuples $\left(z_{1}, \cdots, z_{N}\right)$ with at least one negative element, decreases exponentially with $\rho$ and therefore can be neglected compared to $P_{O^{+}}$which decreases polynomially with $\rho$.

\section{DIVERSITY-MULTIPLEXING TRADEOFF ANALYSIS}

In non-ergodic fading channels, performance of the connection is evaluated in terms of outage probability, which is defined as the event that the instantaneous mutual information does not support the intended rate [23], [24], i.e.,

$$
O_{p} \triangleq\{\mathbf{H} \mid I(x ; y \mid \boldsymbol{H}=\mathbf{H})<R\}
$$

where $\mathbf{H}$ is a channel realization and $R$ is the transmission rate. The lower bound of $O_{p}$ 's probability is defined as outage probability $P_{o}(R, \rho)$ [23], [24]. Therefore,

$$
\begin{aligned}
P_{o}(R, \rho) & =\inf _{\boldsymbol{\Lambda}_{\mathrm{x}}} \operatorname{Pr}\left\{O_{p}\right\} \\
& =\operatorname{Pr}\left\{\max _{\boldsymbol{\Lambda}_{\mathrm{x}}} I(x ; y \mid \mathbf{H})<R\right\} .
\end{aligned}
$$

We measure the system performance by the system outage probability (SOP). SOP is the probability of the system outage event (SOE) which occurs if the capacity of any $s \rightarrow d$ link is less than the transmission rate of $s_{k}$. We assume that the two sources have the same data rate $R$. Correspondingly, we define the system frame error probability (SFEP), which means that a frame is successfully transmitted if and only if both destinations can successfully receive the frame. The system diversity gain is defined as

$$
d^{*}(\lambda)=\frac{\log P_{e, \mathrm{sys}}}{\log \rho}
$$

where $P_{e, \text { sys }}$ is the SFEP. Then, we turn to each network coding protocol to give the DMT analysis.

\section{A. NRNC Protocol}

Denote $O_{d_{k}}$ as the outage event of $d_{k}$ and then the system outage event is expressed as

$$
O_{\mathrm{sys}}^{\mathrm{NRNC}}=O_{d_{1}} \cup O_{d_{2}} .
$$

So the system outage probability is

$$
\begin{aligned}
P_{o, \mathrm{sys}}^{\mathrm{NRNC}} & =P_{o, d_{1}}+P_{o, d_{2}}-P_{o, d_{1}} P_{o, d_{2}} \\
& \doteq P_{o, d_{1}}+P_{o, d_{2}}
\end{aligned}
$$

where $P_{o, d_{k}}$ is the probability of $O_{d_{k}}$. Since the $\left\{s_{1}, s_{2}\right\} \rightarrow d_{k}$ link is a multi-access channel, $O_{d_{k}}$ is formalized as

$$
\begin{aligned}
O_{d_{k}}= & \left\{\mathbf{H} \mid I\left(\mathbf{x}_{s} ; \mathbf{y}_{d_{k}} \mid \mathbf{H}\right)<2 N R,\right. \\
& \left.I\left(\mathbf{x}_{s_{1}} ; \mathbf{y}_{d_{k}} \mid \mathbf{H}\right)<N R, I\left(\mathbf{x}_{s_{2}} ; \mathbf{y}_{d_{k}} \mid \mathbf{H}\right)<N R\right\} .
\end{aligned}
$$

When outage region is determined, $P_{o, d_{k}}$ can be obtained by (10) if $\rho$ is large enough. On the other hand, the SFEP of the NRNC protocol is

$$
\begin{aligned}
P_{e, \mathrm{sys}}^{\mathrm{NRNC}} & =P_{e, d_{1}}+P_{e, d_{2}}-P_{e, d_{1}} P_{e, d_{2}} \\
& \doteq P_{e, d_{1}}+P_{e, d_{2}}
\end{aligned}
$$

where $P_{e, d_{k}}$ is the error decoding probability of a system frame in the $k$ th destination and its lower bound is $P_{o, d_{k}}$. Then, by developing the method in [19], we get the following theorem.

Theorem 1: The DMT of NRNC protocol is given by

$$
d^{*}(\lambda)=(1-\lambda)^{+} .
$$

Proof: See Appendix I.

\section{B. RCNC Protocol}

In RCNC, system outage event is

$$
O_{\mathrm{sys}}^{\mathrm{RCNC}}=O_{r} \cup O_{d_{1} \mid r} \cup O_{d_{2} \mid r} .
$$

So the system outage probability is

$$
P_{o, \text { sys }}^{\mathrm{RCNC}} \doteq P_{o, r}+P_{o, d_{1} \mid r}+P_{o, d_{2} \mid r}
$$

where $P_{o, r}$ is the probability of $O_{r}$ which denotes the outage event at the relay and $P_{o, d_{k} \mid r}$ is the probability of $O_{d_{k} \mid r}$ which denotes the outage event at the $k$ th destination on the condition 


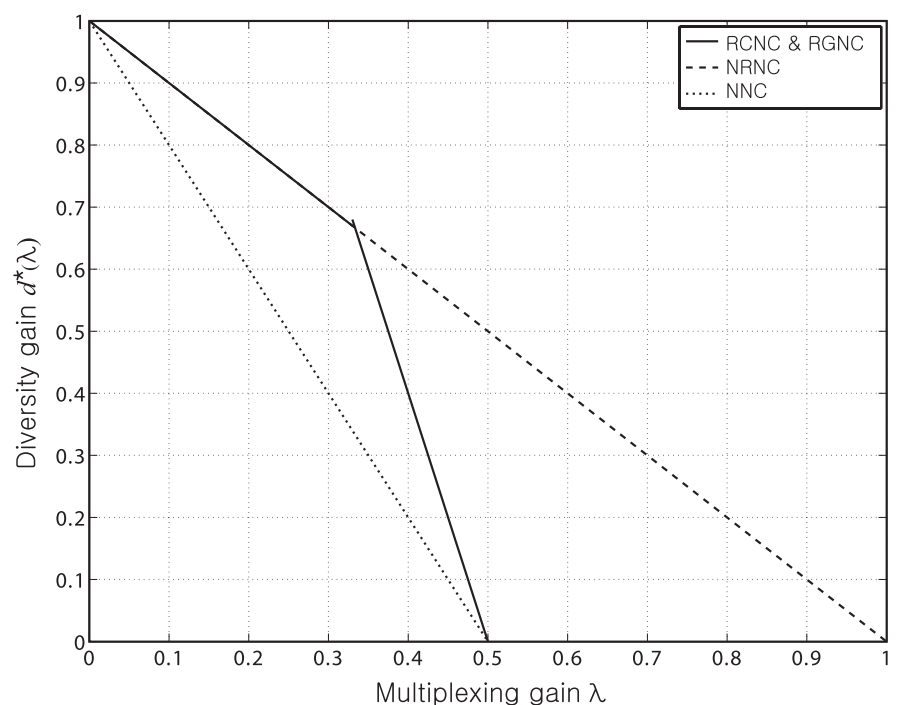

Fig. 3. DMT curves of 4 transmission schemes.

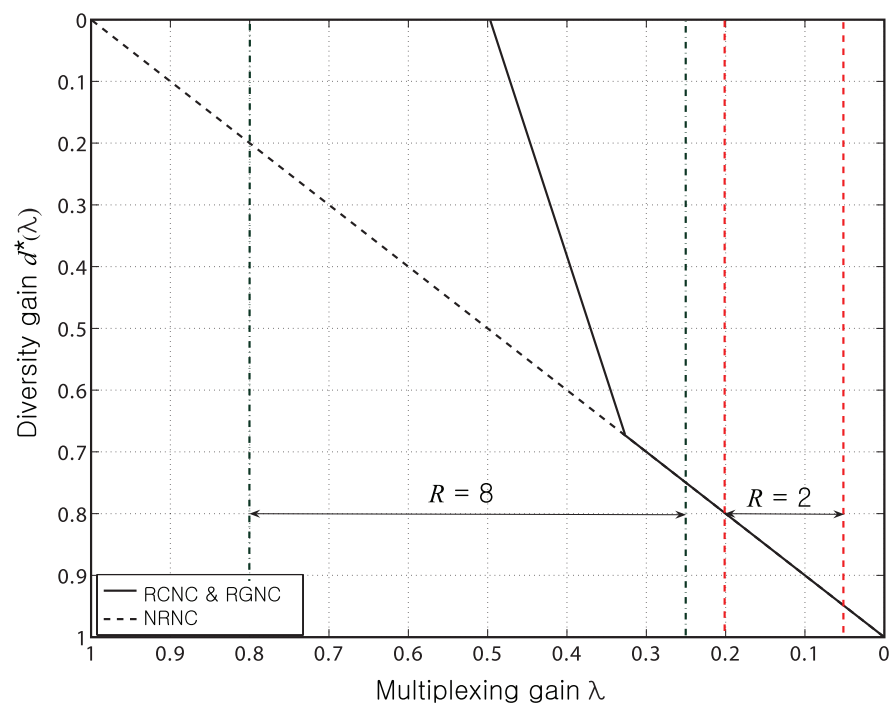

Fig. 4. Modified DMT curves which give a better understanding of the SOP curves. We consider the transmission rate $R=2$ and $R=8$ with $\rho$ 's variation from $30 \mathrm{~dB}$ to $90 \mathrm{~dB}$. Then, we can compute the multiplexing gain regions as $0.0669 \leq \lambda \leq 0.2006$ and $0.2676 \leq \lambda \leq$ 0.8024 for $R=2$ and $R=8$, respectively. DMT predicts the diversity gain of the SOP curves in these multiplexing gain regions by linear approximation.

that $r$ has successfully decoded $\mathbf{x}_{s}$. Since $\left\{s_{1}, s_{2}\right\} \rightarrow r$ link is a multi-access channel, $O_{r}$ is formalized as

$$
\begin{aligned}
& O_{r}=\{\mathbf{H} \mid I\left(\mathbf{x}_{s} ; \mathbf{y}_{r} \mid \mathbf{H}\right)<2 N R, \\
&\left.I\left(\mathbf{x}_{s_{1}} ; \mathbf{y}_{r} \mid \mathbf{H}\right)<N R, I\left(\mathbf{x}_{s_{2}} ; \mathbf{y}_{r} \mid \mathbf{H}\right)<N R\right\}
\end{aligned}
$$

where $\mathbf{y}_{r}$ is the signals received by the relay. Note that $\left\{s_{k}, r\right\} \rightarrow d_{k}$ link is also a multi-access channel. Then, we focus on $d_{1}$ and get that

$$
\begin{aligned}
& O_{d_{1} \mid r}=\left\{\mathbf{H} \mid I\left(\mathbf{x}_{s_{1}}, \mathbf{x}_{r}^{c} ; \mathbf{y}_{d_{1}} \mid \mathbf{H}\right)<2 N R,\right. \\
& \left.I\left(\mathbf{x}_{s_{1}}, \mathbf{x}_{r_{1}} ; \mathbf{y}_{d_{1}} \mid \mathbf{H}\right)<N R, I\left(\mathbf{x}_{r_{2}} ; \mathbf{y}_{d_{1}} \mid \mathbf{H}\right)<N R\right\} .
\end{aligned}
$$

On the other hand, the SFEP of RCNC is

$$
P_{e, \mathrm{sys}}^{\mathrm{RCNC}} \doteq P_{e, r}+P_{e, d_{1} \mid r}+P_{e, d_{2} \mid r}
$$

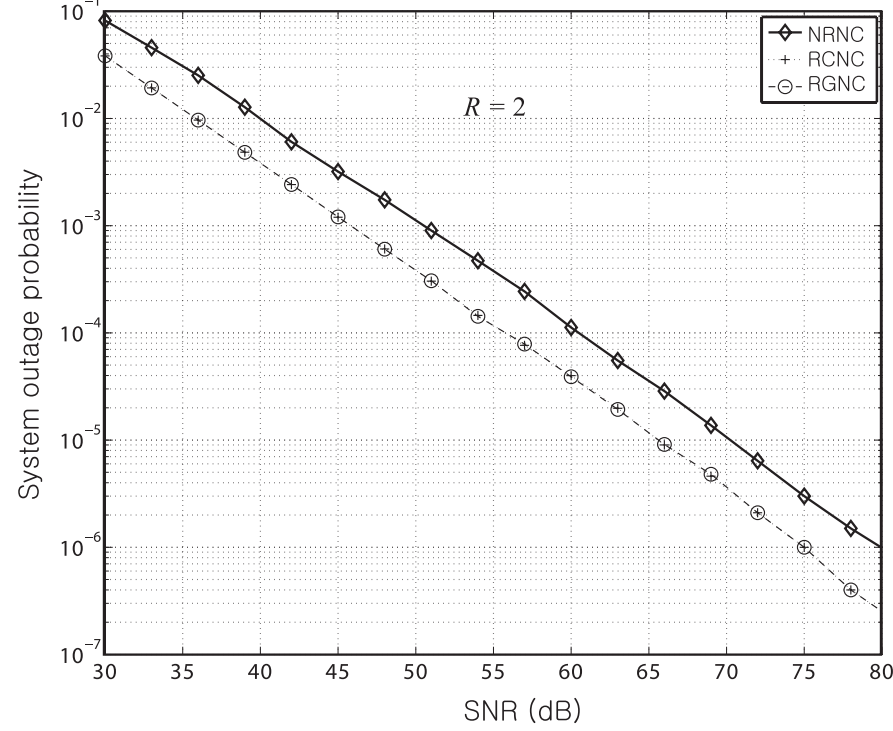

Fig. 5. SOP curves of the three network coding protocols when $R=2$.

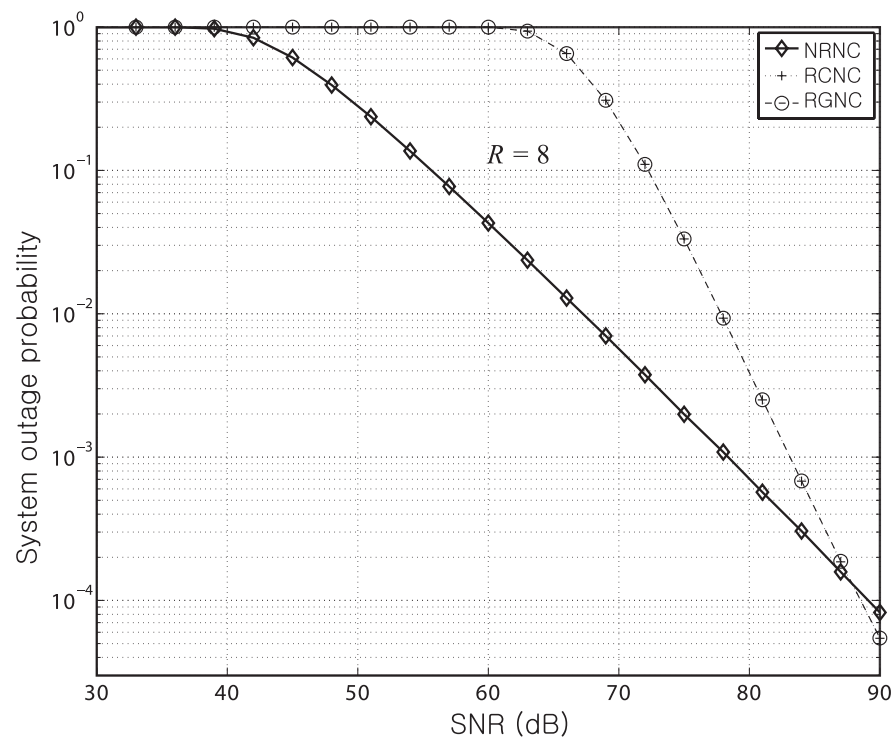

Fig. 6. SOP curves of the three network coding protocols when $R=8$.

where $P_{e, r}$ is the error decoding probability of a system frame in the relay and $P_{e, d_{k} \mid r}$ is the error decoding probability of a system frame on the condition that $r$ has successfully decoded $\mathbf{x}_{s}$ in the $k$ th destination. The DMT formulation is then given by the following theorem.

Theorem 2: The DMT of RCNC is given by

$$
d^{*}(\lambda)= \begin{cases}1-\lambda & \text { for } \lambda \leq \frac{1}{3} \\ 2-4 \lambda & \text { for } \frac{1}{3}<\lambda \leq \frac{1}{2} \\ 0 & \text { for } \frac{1}{2}<\lambda .\end{cases}
$$

Proof: See Appendix II.

\section{RGNC Protocol}

In RGNC, system outage event is

$$
O_{\text {sys }}^{\mathrm{RGNC}}=O_{r} \cup O_{d_{1} \mid r} \cup O_{d_{2} \mid r} .
$$


So the system outage probability is

$$
P_{o, \text { sys }}^{\mathrm{RGNC}} \doteq P_{o, r}+P_{o, d_{1} \mid r}+P_{o, d_{2} \mid r}
$$

where $P_{o, r}$ is the probability of $O_{r}$ which denotes the outage event at the relay and $P_{o, d_{k} \mid r}$ is the probability of $O_{d_{k} \mid r}$ which denotes the outage event at the $k$ th destination on the condition that $r$ has successfully decoded $\mathbf{x}_{s}$.

Obviously, $O_{r}$ of RGNC protocol is equal to that of RCNC protocol. However, $O_{d_{k} \mid r}$ in the two protocols are different. We focus on $d_{1}$ and get

$$
O_{d_{1} \mid r}=\left\{\mathbf{H} \mid I\left(\mathbf{x}_{s_{1}} ; \mathbf{y}_{d_{1}} \mid \mathbf{H}\right)<N R,\left(\mathbf{x}_{r}^{g} ; \mathbf{y}_{d_{1}} \mid \mathbf{H}\right)<N R\right\} .
$$

Note that

$$
P_{e, \mathrm{sys}}^{\mathrm{RCNC}} \doteq P_{e, r}+P_{e, d_{1} \mid r}+P_{e, d_{2} \mid r}
$$

where $P_{e, r}$ and $P_{e, d_{k} \mid r}$ have the same definitions as that in RCNC. Then, the DMT formulation is given by the following theorem.

Theorem 3: The DMT of RCNC is given by

$$
d^{*}(\lambda)= \begin{cases}1-\lambda & \text { for } \lambda \leq \frac{1}{3} \\ 2-4 \lambda & \text { for } \frac{1}{3}<\lambda \leq \frac{1}{2} \\ 0 & \text { for } \frac{1}{2}<\lambda\end{cases}
$$

Proof: It is straightforward to see $P_{o, r}$ in the RCNC and RGNC are equal. According to Appendix II,

$$
d^{*}(\lambda)= \begin{cases}1-\lambda & \text { for } \lambda \leq \frac{1}{3} \\ 2-4 \lambda & \text { for } \frac{1}{3}<\lambda \leq \frac{1}{2} \\ 0 & \text { for } \frac{1}{2}<\lambda\end{cases}
$$

Then, we turn to $P_{o, d_{1} \mid r}$ and easily get

$$
P_{o, d_{1} \mid r} \doteq \rho^{-d_{o, d_{1}}} \quad \text { where } \quad d_{o, d_{1}}=(1-\lambda)^{+} .
$$

Combine (30) and (31), and we get

$$
P_{o, \text { sys }}^{\mathrm{RGNC}} \doteq \rho^{-d_{o, r}}
$$

where $d_{o, r}$ provides the upper bound of $d^{*}(\lambda)$. On the other hand, we can select a large enough frame length $N$ to achieve this bound.

\section{NUMERICAL RESULTS}

Since the wireless network coding can enhance the system throughput, we then give a comparison with the traditional transmission scheme from the view of DMT. As mentioned in Section I, the traditional scheme uses $4 N$ time slots to transmit the $2 N$ symbols in orthogonal time division channels. So the multiplexing gain is no larger than $\frac{1}{2}$. Meanwhile, its maximum diversity gain is 1 since the worst link, i.e., $s_{k} \rightarrow d_{\bar{k}}$ where $\bar{k}$ is the complementary element of $k$ in set $\{1,2\}$, only has one diversity order. Fig. 3 illuminates the DMT of different transmission schemes, we can see that the three network coding protocols have the same DMT formulations when $\lambda \leq \frac{1}{3}$. As $\lambda$ increases, the system diversity gain decreases faster in RCNC and
RGNC than that in NRNC, which means that if the multiplexing gain $\lambda$ is low, the three protocols have the same system performance in the sense of diversity gain, while $\lambda$ is high enough, the performance of the two regenerative network coding (RNC) protocols deteriorates more than that of NRNC. This is due to that the relay in the two regenerative network coding protocols should deal with the multi-access channels and decode the symbols from both sources. On the other hand, the traditional transmission scheme without network coding (NC) has the least multiplexing gain compared to the three network coding protocols under the same system performance.

Since DMT provides the diversity gain under the fixed $R$ and $\rho$, it can predict the SOP curves by asymptotic way. According to the three theorems, we focus on the two kinds of multiplexing gain region, i.e., the lower multiplexing gain region $\left(\lambda<\frac{1}{3}\right)$ and higher multiplexing gain region $\left(\lambda \geq \frac{1}{3}\right)$. To construct the two regions, we select the transmission rate as $R=2$ and $R=8$ and let $\rho$ cover from $30 \mathrm{~dB}$ to $90 \mathrm{~dB}$. Then, we get two kinds of multiplexing gain region as $0.0669 \leq \lambda \leq 0.2006$ and $0.2676 \leq$ $\lambda \leq 0.8024$ (see Fig. 4)

We first focus on the lower multiplexing gain region where $R=2$ and $0.0669 \leq \lambda \leq 0.2006$. Since $\lambda \leq \frac{1}{3}$, DMT predicts that the three protocols have the same diversity gain $d^{*}(\lambda)=1$. Monte-Carlo simulations in Fig. 5 verify the prediction, i.e., the three protocols have the same diversity gain. Note that the gap of the SOP curves between NRNC and RCNC (RGNC as well) is due to the different coding gain, i.e., the two regenerative network coding protocols achieve better coding gain than NRNC. This gap can be neglected when compared with the diversity gain if $\rho$ is large enough. On the other hand, in the higher multiplexing gain region where $R=8$ and $0.2676 \leq \lambda \leq 0.8024$, DMT predicts that NRNC has a better performance than the two regenerative network coding protocols due to the higher diversity gain. Monte-Carlo simulation in Fig. 6 verifies the prediction.

\section{CONCLUSION}

This paper proposes three network coding protocols and gives the DMT analysis for the protocols. The DMT curves show that the three protocols outperform the traditional transmission scheme to have more system multiplexing gain under the same system performance (or diversity gain). On the other hand, we turn to the system performance gain under the fixed multiplexing gain. When at a lower multiplexing gain region, i.e., $\lambda \leq \frac{1}{3}$, the three protocols have the same system performance. However, when $\lambda>\frac{1}{3}$, NRNC beats the other two protocols and owns the best performance. Monte-Carlo simulations prove the conjectures of DMT. In this sense, DMT can asymptotically predict the SOP curves and gives a fast and effective understanding of the system performance.

\section{APPENDICES}

\section{PROOF OF THEOREM 1}

Since SOP is the lower bound of SFEP, we first deduce SOP and give the upper bound of the system diversity gain, following which, we prove that this bound is achievable. 


\section{A. Upper Bound}

Without loss of generality, we focus on $d_{1}$ and rewrite (3) in matrix form as

$\mathbf{y}_{d_{1}}=\left[\begin{array}{cc}\hbar_{1} \mathbf{I}_{N} & \mathbf{O}_{N} \\ g_{1} b h_{1} \mathbf{I}_{N} & g_{2} b h_{1} \mathbf{I}_{N}\end{array}\right] \mathbf{K}_{2 N} \mathbf{x}_{s}+\left[\begin{array}{cc}\mathbf{O}_{N} & \mathbf{O}_{N} \\ \mathbf{O}_{N} & b h_{1} \mathbf{I}_{N}\end{array}\right] \mathbf{v}_{r}+\mathbf{v}_{d_{1}}$

where $\mathbf{O}_{N}$ and $\mathbf{I}_{N}$ are the $N$-order zero and unit matrix, respectively, $\mathbf{K}_{2 N}=\operatorname{diag}\left(\sqrt{\kappa_{1}}, \cdots, \sqrt{\kappa_{1}}, \sqrt{\kappa_{2}}, \cdots, \sqrt{\kappa_{2}}\right)$ is the power allocation matrix, $\mathbf{v}_{r}=\left[0, \cdots, 0, v_{r, 1}, \cdots, v_{r_{N}}\right]^{T}$ is the noise vector observed by the relay, and $\mathbf{v}_{d_{1}}=\left[v_{d_{1}, 1}, \cdots, v_{d_{1}, 2 N}\right]^{T}$ is the noise vector observed by $d_{1}$. Consider the channels are static during a frame period and all symbols are i.i.d. Then,

$$
I\left(\mathbf{x}_{s} ; \mathbf{y}_{d_{1}}\right)=\sum_{i=1}^{N} I\left(\mathbf{x}_{s}^{i} ; \mathbf{y}_{d_{1}}^{i}\right)=N I\left(\mathbf{x}_{s}^{i} ; \mathbf{y}_{d_{1}}^{i}\right)
$$

where $\mathbf{x}_{s}^{i}=\left[\sqrt{\kappa_{1}} x_{s_{1}, i}, \sqrt{\kappa_{2}} x_{s_{2}, i}\right]^{T}, \mathbf{y}_{d_{1}}^{i}=\mathbf{G}_{i} \mathbf{x}_{s}^{i}+\mathbf{v}_{i}$, and

$$
\mathbf{G}_{i}=\left[\begin{array}{cc}
\hbar_{1} & 0 \\
g_{1} b h_{1} & g_{2} b h_{1}
\end{array}\right], \mathbf{v}_{i}=\left[\begin{array}{c}
v_{d_{1}, i} \\
b h_{1} v_{r, i}+v_{d_{1}, N+i}
\end{array}\right] .
$$

The mutual information between $\mathbf{x}_{s}^{i}$ and $\mathbf{y}_{d_{1}}^{i}$ is given by

$$
I\left(\mathbf{x}_{s}^{i} ; \mathbf{y}_{d_{1}}^{i}\right)=\log \left(\operatorname{det}\left(\mathbf{I}_{2}+\boldsymbol{\Lambda}_{\mathbf{v}_{i}}^{-\frac{1}{2}} \mathbf{G}_{i} \boldsymbol{\Lambda}_{\mathbf{x}_{s}^{i}} \mathbf{G}_{i}^{H} \boldsymbol{\Lambda}_{\mathbf{v}_{i}}^{-\frac{1}{2}}\right)\right) .
$$

A lower bound of $\max _{\Lambda_{\mathbf{x}_{s}^{i}}} I\left(\mathbf{x}_{s}^{i} ; \mathbf{y}_{d_{1}}^{i}\right)$ is obtained if power are equally allocated to each transmitter, i.e.,

$$
\log \left(\operatorname{det}\left(\mathbf{I}_{2}+\frac{2 P}{3} \mathbf{G}_{i} \mathbf{G}_{i}^{H} \boldsymbol{\Lambda}_{\mathbf{v}_{i}}^{-1}\right)\right) \leq \max _{\boldsymbol{\Lambda}_{\mathbf{x}_{s}^{i}}} I\left(\mathbf{x}_{s}^{i} ; \mathbf{y}_{d_{1}}^{i}\right) \text {. }
$$

On the other hand,

$$
\max _{\boldsymbol{\Lambda}_{\mathbf{x}_{s}^{i}}} I\left(\mathbf{x}_{s}^{i} ; \mathbf{y}_{d_{1}}^{i}\right)<\log \left(\operatorname{det}\left(\mathbf{I}_{2}+2 P \mathbf{G}_{i} \mathbf{G}_{i}^{H} \boldsymbol{\Lambda}_{\mathbf{v}_{i}}^{-1}\right)\right) .
$$

The two bounds converge as $\rho$ grows to infinity, i.e.,

$$
\begin{aligned}
& \lim _{\rho \rightarrow \infty} \frac{\max _{\mathbf{x}_{s}^{j}} I\left(\mathbf{x}_{s}^{i} ; \mathbf{y}_{d_{1}}^{i}\right)}{\log \rho}= \\
& \lim _{\rho \rightarrow \infty} \frac{\log \left(\operatorname{det}\left(\mathbf{I}_{2}+P \mathbf{G}_{i} \mathbf{G}_{i}^{H} \mathbf{\Lambda}_{\mathbf{v}_{i}}^{-1}\right)\right)}{\log \rho} .
\end{aligned}
$$

Plugging in for $\mathbf{G}_{i}$ and $\mathbf{v}_{i}$, we get

$$
\begin{aligned}
\lim _{\rho \rightarrow \infty} & \frac{\max _{\mathbf{x}_{s}^{i}} I\left(\mathbf{x}_{s}^{i} ; \mathbf{y}_{d_{1}}^{i}\right)}{\log \rho}=\lim _{\rho \rightarrow \infty} \frac{1}{\log \rho} \log \left(1+\left|\hbar_{1}\right|^{2} \rho+\right. \\
& \left.\frac{\left(\left|g_{1}\right|^{2}+\left|g_{2}\right|^{2}\right)\left|h_{1}\right|^{2} b^{2} \rho}{1+\left|h_{1}\right|^{2} b^{2}}+\frac{\left|g_{2}\right|^{2}\left|\hbar_{1}\right|^{2}\left|h_{1}\right|^{2} b^{2} \rho^{2}}{1+\left|h_{1}\right|^{2} b^{2}}\right) .
\end{aligned}
$$

Similarly, we can get

$$
\begin{aligned}
& \lim _{\rho \rightarrow \infty} \frac{\max I\left(x_{s_{2}, i} ; \mathbf{y}_{d_{1}}^{i}\right)}{\log \rho}=\lim _{\rho \rightarrow \infty} \frac{1}{\log \rho} \log \left(1+\frac{\left|g_{2}\right|^{2}\left|h_{1}\right|^{2} b^{2} \rho}{1+\left|h_{1}\right|^{2} b^{2}}\right) \\
& \lim _{\rho \rightarrow \infty} \frac{\max I\left(x_{s_{1}, i} ; \mathbf{y}_{d_{1}}^{i}\right)}{\log \rho}= \\
& \lim _{\rho \rightarrow \infty} \frac{1}{\log \rho} \log \left(1+\left|\hbar_{1}\right|^{2} \rho+\frac{\left|g_{1}\right|^{2}\left|h_{1}\right|^{2} b^{2} \rho}{1+\left|h_{1}\right|^{2} b^{2}}\right) .
\end{aligned}
$$

It is straightforward to see that

$$
\begin{aligned}
& \lim _{\rho \rightarrow \infty} \frac{\max I\left(x_{s_{1}, i} ; \mathbf{y}_{d_{1}}^{i}\right)}{\log \rho}=\left(\max \left\{1-z_{\hbar_{1}}, 1-\left(z_{g_{1}}+z_{h_{1}}\right)\right\}\right)^{+}, \\
& \lim _{\rho \rightarrow \infty} \frac{\max I\left(x_{s_{2}, i} ; \mathbf{y}_{d_{1}}^{i}\right)}{\log \rho}=\left(1-\left(z_{g_{2}}+z_{h_{1}}\right)\right)^{+}, \\
& \lim _{\rho \rightarrow \infty} \frac{\max _{\mathbf{x}_{s}^{i}} I\left(\mathbf{x}_{s}^{i} ; \mathbf{y}_{d_{1}}^{i}\right)}{\log \rho}=\left(\operatorname { m a x } \left\{1-z_{\hbar_{1}}, 1-\left(z_{g_{1}}+z_{h_{1}}\right),\right.\right. \\
& \left.\left.1-\left(z_{g_{2}}+z_{h_{1}}\right), 2-\left(z_{g_{2}}+z_{h_{1}}+z_{\hbar_{1}}\right)\right\}\right)^{+}
\end{aligned}
$$

where $z$ with different subscripts are similar to (7) and denote the negative order of corresponding channels. According to the outage region $O_{d_{1}}$ determined by (16), we conclude that

$$
O_{d_{1}}=O_{d_{1}}^{\mathbf{x}_{s_{1}}} \cup O_{d_{1}}^{\mathbf{x}_{s_{2}}} \cup O_{d_{1}}^{\mathbf{x}_{s}}
$$

where $O_{d_{1}}^{\mathbf{x}_{s_{1}}}\left(O_{d_{1}}^{\mathbf{x}_{s_{2}}}\right)$ represents the outage event which occurs only between $s_{1}\left(s_{2}\right)$ and $d_{1}$ and $O_{d_{1}}^{\mathbf{x}_{s}}$ represents the outage event which occurs between both sources and $d_{1}$. Thus,

$$
\begin{aligned}
P_{o, d_{1}} & =P\left(O_{d_{1}}^{\mathbf{x}_{s_{1}}}\right)+P\left(O_{d_{1}}^{\mathbf{x}_{s_{2}}}\right)+P\left(O_{d_{1}}^{\mathbf{x}_{s}}\right) \\
& \doteq \max \left\{P\left(O_{d_{1}}^{\mathbf{x}_{s_{1}}}\right), P\left(O_{d_{1}}^{\mathbf{x}_{s_{2}}}\right), P\left(O_{d_{1}}^{\mathbf{x}_{s}}\right)\right\} .
\end{aligned}
$$

From (42), the outage regions are

$$
\begin{aligned}
& O_{d_{1}}^{\mathbf{x}_{s_{1}}}=\left\{\left(z_{\hbar_{1}}, z_{g_{1}}, z_{h_{1}}\right) \in R^{3+} \mid\right. \\
&\left.\max \left\{1-z_{\hbar_{1}}, 1-\left(z_{g_{1}}+z_{h_{1}}\right)\right\}<\lambda\right\}, \\
& O_{d_{1}}^{\mathbf{x}_{s_{2}}}=\left\{\left(z_{g_{2}}, z_{h_{1}}\right) \in R^{2+} \mid 1-\left(z_{g_{2}}+z_{h_{1}}\right)<\lambda\right\}, \\
& O_{d_{1}}^{\mathbf{x}_{s}}=\left\{\left(z_{\hbar_{1}}, z_{g_{1}}, z_{g_{2}}, z_{h_{1}}\right) \in R^{4+} \mid\right. \\
& \max \left\{1-z_{\hbar_{1}}, 1-\left(z_{g_{1}}+z_{h_{1}}\right),\right. \\
&\left.\left.1-\left(z_{g_{2}}+z_{h_{1}}\right), 2-\left(z_{g_{2}}+z_{h_{1}}+z_{\hbar_{1}}\right)\right\}<2 \lambda\right\} .
\end{aligned}
$$

According to (44), we pick up the maximum among the three elements, which means that we should find out

$$
\begin{array}{r}
\min \left\{\inf _{O_{d_{1}}^{\mathbf{x}_{s_{1}}}}\left(z_{\hbar_{1}}+z_{g_{1}}+z_{h_{1}}\right), \inf _{O_{\mathbf{x}_{1}}^{\mathbf{x}_{s_{2}}}}\left(z_{g_{2}}+z_{h_{1}}\right),\right. \\
\left.\inf _{O_{d_{1}}^{\mathbf{x}_{s}}}\left(z_{\hbar_{1}}+z_{g_{1}}+z_{g_{2}}+z_{h_{1}}\right)\right\} .
\end{array}
$$

Then, we can easily get that the second item in (46) is the minimum. So

$$
P_{o, d_{1}} \doteq \rho^{-d_{o, d_{1}}} \quad \text { where } \quad d_{o, d_{1}}=(1-\lambda)^{+} .
$$

Due to the symmetry of the system,

$$
P_{o, \text { sys }}^{\mathrm{NRNC}}=2 \rho^{-d_{o, d_{1}}} \doteq \rho^{-d_{o, d_{1}}}
$$

where $d_{o, d_{1}}$ provides the upper bound of $d^{*}(\lambda)$ of NRNC. 


\section{B. Achievability}

According to the definition of SFEP,

$$
\begin{aligned}
P_{e, \mathrm{sys}}^{\mathrm{NRNC}} & =P_{e, d_{1}}+P_{e, d_{2}}-P_{e, d_{1}} P_{e, d_{2}} \\
& \doteq P_{e, d_{1}}+P_{e, d_{2}} .
\end{aligned}
$$

Without loss of generality, we focus on $P_{e, d_{1}}$ and define that

$$
E_{d_{1}}=E_{d_{1}}^{\mathbf{x}_{s_{1}}} \cup E_{d_{1}}^{\mathbf{x}_{s_{2}}} \cup E_{d_{1}}^{\mathbf{x}_{s}}
$$

where $E_{d_{1}}^{\mathbf{x}_{s_{1}}}\left(E_{d_{1}}^{\mathbf{x}_{s_{2}}}\right)$ represents the error event in $d_{1}$ that only $s_{1}\left(s_{2}\right)$ is detected in error and $E_{d_{1}}^{\mathbf{x}_{s}}$ represents the error event in $d_{1}$ that both sources are detected in error. Thus,

$$
P_{e, d_{1}} \doteq \max \left\{P\left(E_{d_{1}}^{\mathbf{x}_{s_{1}}}\right), P\left(E_{d_{1}}^{\mathbf{x}_{s_{2}}}\right), P\left(E_{d_{1}}^{\mathbf{x}_{s}}\right)\right\}
$$

The decode error probability $P_{e}$ of the ML decoder can be upper bounded using Bayes's rule, i.e., $P_{e}=P_{o}+P_{e, \bar{o}}$ where $P_{o}$ is the outage probability and $P_{e, \bar{o}}$ is the decoding error probability when there is no outage. On the other hand, from [19], the pairwise error probability (PEP) of the ML decoder is upper bounded by

$$
P_{P E} \leq \operatorname{det}\left(I+\frac{1}{2} \boldsymbol{\Lambda}_{\mathbf{x}_{s}} \boldsymbol{\Lambda}_{\mathbf{v}}^{-1}\right)^{-1}
$$

So we conclude that

$$
\begin{aligned}
P\left(E_{d_{1}}^{\mathbf{x}_{s_{1}}}\right) & \leq\left(1+\frac{1}{2}\left|\hbar_{1}\right|^{2} \rho+\frac{1}{2} \frac{\left|g_{1}\right|^{2}\left|h_{1}\right|^{2} b^{2} \rho}{1+\left|h_{1}\right|^{2} b^{2}}\right)^{-N} \rho^{r N}, \\
P\left(E_{d_{1}}^{\mathbf{x}_{s_{2}}}\right) & \leq\left(1+\frac{1}{2} \frac{\left|g_{2}\right|^{2}\left|h_{1}\right|^{2} b^{2} \rho}{1+\left|h_{1}\right|^{2} b^{2}}\right)^{-N} \rho^{r N}, \\
P\left(E_{d_{1}}^{\mathbf{x}_{s}}\right) & \leq\left(1+\frac{1}{2}\left|\hbar_{1}\right|^{2} \rho+\frac{1}{2} \frac{\left(\left|g_{1}\right|^{2}+\left|g_{2}\right|^{2}\right)\left|h_{1}\right|^{2} b^{2} \rho}{1+\left|h_{1}\right|^{2} b^{2}}\right. \\
& \left.+\frac{1}{4} \frac{\left|g_{2}\right|^{2}\left|\hbar_{1}\right|^{2}\left|h_{1}\right|^{2} b^{2} \rho^{2}}{1+\left|h_{1}\right|^{2} b^{2}}\right)^{-2 N} \rho^{2 r N} .
\end{aligned}
$$

When $\rho$ is large enough, $E_{d_{1}}^{\mathbf{x}_{s_{2}}}$ happens with the most probability. So $P_{e, d_{1}} \doteq P_{E_{d_{1}}^{\mathbf{x}_{s_{2}}}}$. From (52), when in the critical region that $O_{d_{1}}^{\mathbf{x}_{s_{2}}}$ not happens, i.e., $1-\left(z_{g_{2}}+z_{h_{1}}\right)=\lambda$, we make the frame length so long that it can make $P_{E_{d_{1}, \bar{o}}^{\mathbf{x}_{s_{2}}}}$ arbitrarily small where $P_{E_{d_{1}, \bar{o}}^{\mathbf{x}_{s_{2}}}}$ denotes the error decoding probability when there is no outage. Then, we can say that the diversity bound $d_{o, d_{1}}$ is achievable by selecting a large enough frame length $N$ and thus complete the proof.

\section{PROOF OF THEOREM 2}

Similar to Appendix I, we give the upper bound of the system diversity and then prove the achievability.

We first focus on the outage event between two sources and the relay. The relay receive the signals from two sources in the first $N$ time slot, i.e.,

$$
\mathbf{y}_{r}=g_{1} \sqrt{\kappa_{1}} \mathbf{x}_{s_{1}}+g_{2} \sqrt{\kappa_{2}} \mathbf{x}_{s_{2}}+\mathbf{v}_{r}
$$

Since all symbols are i.i.d.,

$$
\begin{aligned}
I\left(\mathbf{x}_{s_{1}}, \mathbf{x}_{s_{2}} ; \mathbf{y}_{r}\right) & =\sum_{i=1}^{N} I\left(x_{s_{1}, i}, x_{s_{2}, i} ; y_{r_{i}}\right) \\
& =N I\left(x_{s_{1}, i}, x_{s_{2}, i} ; y_{r_{i}}\right)
\end{aligned}
$$

According to the outage region $O_{r}$, we conclude that

$$
O_{r}=O_{r}^{\mathbf{x}_{s_{1}}} \cup O_{r}^{\mathbf{x}_{s_{2}}} \cup O_{r}^{\mathbf{x}_{s}}
$$

where $O_{r}^{\mathbf{x}_{s_{1}}}\left(O_{r}^{\mathbf{x}_{s_{2}}}\right)$ represents the outage event which occurs only between $s_{1}\left(s_{2}\right)$ and $r$, and $O_{r}^{\mathbf{x}_{s}}$ represents the outage event which occurs between both sources and $r$. Thus,

$$
\begin{aligned}
P_{o, r} & =P\left(O_{r}^{\mathbf{x}_{s_{1}}}\right)+P\left(O_{r}^{\mathbf{x}_{s_{2}}}\right)+P\left(O_{r}^{\mathbf{x}_{s}}\right) \\
& \doteq \max \left\{P\left(O_{r}^{\mathbf{x}_{s_{1}}}\right), P\left(O_{r}^{\mathbf{x}_{s_{2}}}\right), P\left(O_{r}^{\mathbf{x}_{s}}\right)\right\} .
\end{aligned}
$$

When $\rho$ is large enough, the outage regions are approximatively defined as if each node consume the same power $P$,

$$
\begin{aligned}
& O_{r}^{\mathbf{x}_{s_{1}}}=\left\{z_{g_{1}} \in R^{+} \mid 1-z_{g_{1}}<\lambda\right\}, \\
& O_{r}^{\mathbf{x}_{s_{2}}}=\left\{z_{g_{2}} \in R^{+} \mid 1-z_{g_{2}}<\lambda\right\}, \\
& O_{r}^{\mathbf{x}_{s}}=\left\{\left(z_{g_{1}}, z_{g_{2}}\right) \in R^{2+} \mid \max \left\{1-z_{g_{1}}, 1-z_{g_{2}}\right\}<2 \lambda\right\} .
\end{aligned}
$$

According to (57), we pick up the maximum among the three elements, which means that we should find out

$$
\min \left\{\inf _{O_{r}^{\mathbf{x}_{1}}} z_{g_{1}}, \inf _{O_{r}^{\mathbf{x}_{2}}} z_{g_{2}}, \inf _{O_{r}^{\mathbf{x}_{s}}}\left(z_{g_{1}}+z_{g_{2}}\right)\right\} .
$$

Similar to Appendix I, we can get that $P_{o, r} \doteq \rho^{-d_{o, r}}$ where

$$
d_{o, r}= \begin{cases}1-\lambda & \text { for } \lambda \leq \frac{1}{3} \\ 2-4 \lambda & \text { for } \frac{1}{3}<\lambda \leq \frac{1}{2} \\ 0 & \text { for } \frac{1}{2}<\lambda\end{cases}
$$

Then, we turn to $P_{o, d_{1} \mid r}$. According to the outage region $O_{d_{1} \mid r}$, we conclude that

$$
O_{d_{1} \mid r}=O_{d_{1} \mid r}^{\mathbf{x}_{s_{1}}, \mathbf{x}_{r}^{c}} \cup O_{d_{1} \mid r}^{\mathbf{x}_{s_{1}}, \mathbf{x}_{r_{1}}} \cup O_{d_{1} \mid r}^{\mathbf{x}_{r_{2}}}
$$

where $O_{d_{1} \mid r}^{\mathbf{x}_{s_{1}}, \mathbf{x}_{r_{1}}}\left(O_{d_{1} \mid r}^{\mathbf{x}_{r_{2}}}\right)$ represents the outage event which occurs only between $s_{1}\left(s_{2}\right)$ and $d_{1}$ on the condition that $r$ have already successfully decoding the frame $\mathbf{x}_{s}$ and $O_{d_{1} \mid r}^{\mathbf{x}_{s_{1}}, \mathbf{x}_{r}^{c}}$ represents the outage event which occurs between both sources and $d_{1}$ on the condition that $r$ have already successfully decoding the frame $\mathbf{x}_{s}$. Thus,

$$
\begin{aligned}
P_{o, d_{1} \mid r} & =P\left(O_{d_{1} \mid r}^{\mathbf{x}_{s_{1}}, \mathbf{x}_{r}^{c}}\right)+P\left(O_{d_{1} \mid r}^{\mathbf{x}_{s_{1}}, \mathbf{x}_{r_{1}}}\right)+P\left(O_{d_{1} \mid r}^{\mathbf{x}_{r_{2}}}\right) \\
& \doteq \max \left\{P\left(O_{d_{1} \mid r}^{\mathbf{x}_{s_{1}}, \mathbf{x}_{r}^{c}}\right), P\left(O_{d_{1} \mid r}^{\mathbf{x}_{s_{1}}, \mathbf{x}_{r_{1}}}\right), P\left(O_{d_{1} \mid r}^{\mathbf{x}_{r_{2}}}\right)\right\} .
\end{aligned}
$$

From the definition of $O_{d_{1} \mid r}$ in (22). It is straightforward to see that when $\rho$ is large enough, the outage events between $\mathbf{x}_{r_{2}}$ and $\mathbf{y}_{d_{1}}$ occurs with the most probability. So

$$
P_{o, d_{1} \mid r} \doteq P\left(O_{d_{1} \mid r}^{\mathbf{x}_{r_{2}}}\right)
$$


where

$$
O_{d_{1} \mid r}^{\mathbf{x}_{r_{2}}}=\left\{z_{h_{1}} \in R^{+} \mid 1-z_{h_{1}}<\lambda\right\} .
$$

So the corresponding probability is

$$
P_{o, d_{1} \mid r} \doteq \rho^{-d_{o, d_{1} \mid r}} \quad \text { for } \quad d_{o, d_{1} \mid r}=(1-\lambda)^{+} .
$$

Combine (60) and (65), and then

$$
P_{o, \text { sys }}^{\mathrm{RCNC}} \doteq P_{o, r}+P_{o, d_{1} \mid r}+P_{o, d_{2} \mid r} \doteq P_{o, r} .
$$

So $d_{o, r}$ provides the upper bound of $d^{*}(\lambda)$ of RCNC. On the other hand, similar to the second subsection in Appendix I, we can prove its achievability by selecting a large enough frame length $N$ when $\rho \rightarrow \infty$. Hence we complete the proof.

\section{ACKNOWLEDGMENTS}

The authors would like to thank the anonymous referees for their constructive comments and suggestions to make this paper much improved over the original submission.

\section{REFERENCES}

[1] R. Ahlswede, N. Cai, S.-Y. R. Li, and R. W. Yeung, "Network infomation flow," IEEE Trans. Inf. Theory, vol. 46, no. 4, pp. 1204-1216, July 2000.

[2] S.-Y. R. Li, R. W. Yeung, and N. Cai, "Linear network coding," IEEE Trans. Inf. Theory, vol. 49, no. 2, pp. 371-381, Feb. 2003.

[3] R. Koetter and M. Medard, "An algebraic approach to network coding," IEEE/ACM Trans. Netw., vol. 11, no. 5, pp. 782-795, Oct. 2003

[4] T. Ho, M. Medard, R. Koetter, D. R. Karger, M. Effros, J. Shi, and B. Leong, "A random linear network coding approach to multicast," IEEE Trans. Inf. Theory, vol. 52, no. 10, pp. 4413-4430, Oct. 2006.

[5] T. Ho and D. S. Lun, Network Coding: An Introduction. Cambridge University Press, 2008

[6] S. Zhang, S.-C. Liew, and P. P. Lam, "Hot topic: Physical-layer network coding," in Proc. MobiCom, Los Angeles, CA, Sept. 23-26, 2006, pp. 358-365.

[7] T. Wang and G. B. Giannakis, "High-throughput cooperative communications with complex field network coding," in Proc. CISS, Baltimore, MD, Mar. 14-16, 2007, pp. 253-258.

[8] L. Xiao, T. E. Fuja, J. Kliewer, and D. J. Costello, "A network coding approach to cooperative diversity," IEEE Trans. Inf. Theory, vol. 53, no. 10, pp. 3714-3722, Oct. 2007

[9] P. Popovski and H. Yomo, "Wireless network coding by amplify-andforward for bi-directional traffic flows," IEEE Commun. Lett., vol. 11, no. 1, pp. 16-18, Jan. 2007.

[10] Y. Wu, P. A. Chou, and S. Kung, "Information exchange in wireless network with network coding and physical-layer broadcast," Microsof Reserach, Redmond, WA, Tech. Rep. MSR-TR-2004-78, Aug. 2004.

[11] J. Li and W. Chen, "Complex field network coding for wireless cooperative multicast flows," in Proc. GLOBECOM, Nov. 2008.

[12] J. Li and W. Chen, "Joint power allocation and precoding for network coding based cooperative multicast systems," IEEE Signal Process. Lett., vol. 15 , pp. 817-820, Nov. 2008.

[13] J. Li and W. Chen, "Power allocation in the high SNR regime for a multicast cell with regenerative network coding," IEEE Commun. Lett., vol. 13, no. 4, pp. 271-273, Apr. 2009.

[14] L. Zheng and D. N. C. Tse, "Diversity and multiplexing: A fundamental tradeoff in multiple antenna channels," IEEE Trans. Inf. Theory, vol. 49, no. 5, pp. 1073-1096, May 2003.

[15] J. N. Laneman, D. N. C. Tse, and G. W. Wornell, "Cooperative diversity in wireless networks: Efficient protocols and outage behavior," IEEE Trans. Inf. Theory, vol. 51, no. 12, pp. 3062-3080, Dec. 2004.
[16] R. U. Nabar, H. Bölcskei, and F. W. Kneubuhler, "Fading relay channels: Performance limits and space-time signal design," IEEE J. Sel. Areas Commun., vol. 22, no. 6, pp. 1099-1109, Aug. 2004.

[17] M. Janani, A. Hedayat, T. Hunter, and A. Nosratinia, "Coded cooperation in wireless communications: Space-tmie transmission and iterative decoding," IEEE Trans. Signal Process., vol. 52, no. 2, pp. 362-371, Feb. 2004.

[18] G. Kramer, M. Gastpar, and P. Gupta, "Cooperative strategies and capacity theorems for relay networks," IEEE Trans. Inf. Theory, vol. 51, no. 9, pp. 3037-3063, Sept. 2005.

[19] K. Azarian, H. El-Gamal, and P. Schniter, "On the achievable diversitymultiplexing tradeoff in half-duplexing cooperative channels," IEEE Trans. Inf. Theory, vol. 51, no. 12, pp. 4152-4172, Dec. 2005.

[20] S. Yang and J.-C. Belfiore, "Towards the optimal amplify-and-forward cooperative diversity scheme," submitted to IEEE Trans. Inf. Theory, Mar. 2006.

[21] M. Yuksel and E. Erkip, "Multiple-antenna cooperative wireless systems: A diversity-multiplexing tradeoff perspective," IEEE Trans. Inf. Theory, vol. 53, no. 10, pp. 3371-3393, Oct. 2007.

[22] H. P. F. Frank and D. K. Marcos, Cooperation in Wireless Networks: Principles and Applications, Netherlands: Springer, 2006.

[23] L. Ozarow, S. Shamai, and A. Wyner, "Information-theoretic considerations in cellular mobile radio," IEEE Trans. Veh. Technol., vol. 43, no. 3, pp. 359-378, May 1994.

[24] İ. E. Telatar, "Capacity of multi-antenna Gaussian channels," Europ. Trans. Telecommun., vol. 10, pp. 585-595, Nov./Dec. 1999.

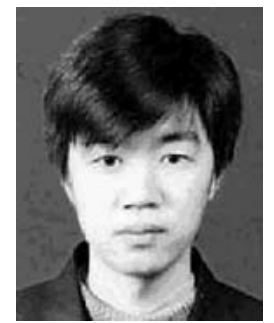

Jun Li was born in 1980. He received the B.S. degree in Electrical Engineering from Jilin University, Changchun, China in 2001, the M.Sc. degree in Electronic Engineering from Nanjing University of Science and Technology, Nanjing, China in 2004, and the Ph.D. degree in Electronic Engineering from Shanghai Jiaotong University in 2009 . He is currently a Technical Staff in Alcatel-bell Technoglogies, Shanghai, China. He is the Technical Program Committee Member for IEEE-ICCSC2008. His interests include network information theory, channel coding theory, and cooperative communications. In this area, he has published 5 papers in IEEE Journals and conferences.

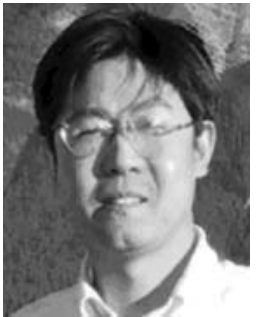

Wen Chen was born in 1967. He received his Ph.D. from University of Electro-Communications, Tokyo, Japan in 1999. He was a JSPS Research Fellow from 1999 through 2001. In 2001, he joined University of Alberta, Canada, starting as a Post-Doctoral Fellow in Information Research Lab and continuing as a Research Associate in Department of Electrical and Computer Engineering. Since 2006, he has been a full Professor in Department of Electronic Engineering, Shanghai Jiaotong University, China, where he is also the Director of Institute for Signal Processing and Systems. He is in the Awarding Committee for Sciences and Technologies, Ministry of Education of China. He was awarded the Ariyama Memorial Research Prize in 1997 and the PIMS Post-Doctoral Fellowship in 2001. He received the honors of "New Century Excellent Young Researcher in China" in 2006 and "the Pujiang Excellent Investigator in Shanghai" in 2007. He is elected the Vice General Secretary of Shanghai Institute of Electronics in 2008. He is in the editorial board of the International Journal of Wireless Communications and Networking and serves Journal of Communications and Journal of Computers as the Guest Editors. He is the General Chair for IEEE-ICIS2009, and the TPC Chair for IEEE-ICCSC2008, ISISE2008, ISCSCT2008, IITS2009, and ISIP2009, a special session Chair for IEEE-SiPS2007, TPC Member for various IEEE conferences. He has published more than 50 papers in IEEE/IEICE journals and conferences. His interests cover cooperative communications and network coding. 